\title{
Proximal and distal spinal muscular atrophy in one family: molecular genetic studies provide further evidence for the non-allelic origin of both diseases
}

Stephanie Spranger, Sabine Rudnik-Schöneborn, Matthias Spranger, Martina Schächtele, Klaus Zerres, Brunhilde Wirth

\begin{abstract}
We present the results of clinical and molecular genetic investigations of a family in which the father suffers from distal spinal muscular atrophy and the younger son is affected by infantile autosomal recessive SMA type I. The molecular analysis of the SMN gene showed homozygous deletions of telSMN exons 7 and 8 in the son only. This was probably the result of a new mutation in the paternal haplotype, since the affected boy did not inherit one copy of the marker Ag1-CA. These results indicate that distal and proximal SMA in this family are not caused by the same gene on chromosome $5 q$. $(千$ Med Genet 1997;34:340-342)
\end{abstract}

Keywords: distal SMA; proximal SMA; spinal muscular atrophy.

Proximal spinal muscular atrophy (SMA) comprises a group of neuromuscular disorders which are characterised by a degeneration of anterior horn cells in the spinal cord leading to symmetrical weakness and wasting of voluntary muscles. Most families with childhood and juvenile onset SMA follow an autosomal recessive pattern of inheritance. Autosomal dominant forms are rare. Three types of autosomal recessive SMA (SMA I-III) have been defined, ${ }^{2}$ depending on the age of onset, motor milestones achieved, and the severity of the clinical course.

After identification of candidate genes, molecular genetic testing in childhood and juvenile onset proximal SMA can be performed. The most important candidate gene is the survival motor neurone (SMN) gene which shows homozygous deletions of the telomeric copy of exons 7 and 8 or exon 7 only in $95-98 \%$ of SMA type I, 95\% of SMA type II, and at least $80-90 \%$ of SMA III patients. ${ }^{3-5}$

Distal SMA is a motor neurone disorder clinically distinct from proximal SMA, as weakness and atrophy predominate in the distal muscles, that is, the hand and foot muscles. The age of onset may vary from childhood to late adulthood, ${ }^{6}$ but the precise molecular basis remains to be identified. Similar to proximal SMA, this is a heterogeneous group comprising autosomal recessive and dominant forms.
One dominant form with onset in adulthood has been localised to chromosome $7 \mathrm{p}^{7}$

The combination of distal and proximal SMA in one family is rare. Three families have been reported with adult onset distal SMA in association with infantile SMA type I in a first or second degree relative. ${ }^{89}$ It has been suggested that distal SMA might be a risk factor for the development of infantile SMA in the offspring. ${ }^{9}$

We report a family with distal SMA in the father and severe infantile SMA in his son, which must be regarded as a coincidence of genetically distinct entities.

\section{Case report}

The pedigree of the family is shown in fig 1 . The index patient is subject III.2. His motor development stopped at the age of 3 months. $\mathrm{He}$ presented with floppiness and absent tendon reflexes. EMG showed signs of denervation and muscle biopsy performed at 7 months showed a mixture of normal and small diameter fibres without inflammation or inclusions, compatible with denervation atrophy. At the age of 2 years, he could lift his head but was unable to turn over or sit unsupported. The clinical picture was classified as SMA I with prolonged survival.

From the age of 30 years his father (subject II.1) had a slowly progressive distal weakness and wasting. Neurological examination at the age of 35 years showed marked weakness in the thenar and interosseous muscles (MRC grade 2) (fig 2). Leg weakness was mild and distally pronounced. He had bilateral pes planus. The EMG showed denervation in the intrinsic hand muscles. Motor and sensory nerve conduction studies were normal. A slowly progressive distal spinal muscular atrophy was diagnosed. The results of neurological examination of his parents (I.1 and I.2), his wife (II.2), and older son (III.1) were normal.

Molecular genetic investigations showed a homozygous deletion of telSMN exons 7 and 8 in patient III. 2 with severe infantile SMA (fig 1), which was not detectable in his father with distal SMA or in other relatives. In addition, an indirect genotype analysis was performed with the multicopy markers $\mathrm{C} 212$ and Ag1-CA which showed evidence of a de novo deletion in the marker Ag1-CA in the patient with SMA I. While the father showed three copies of 


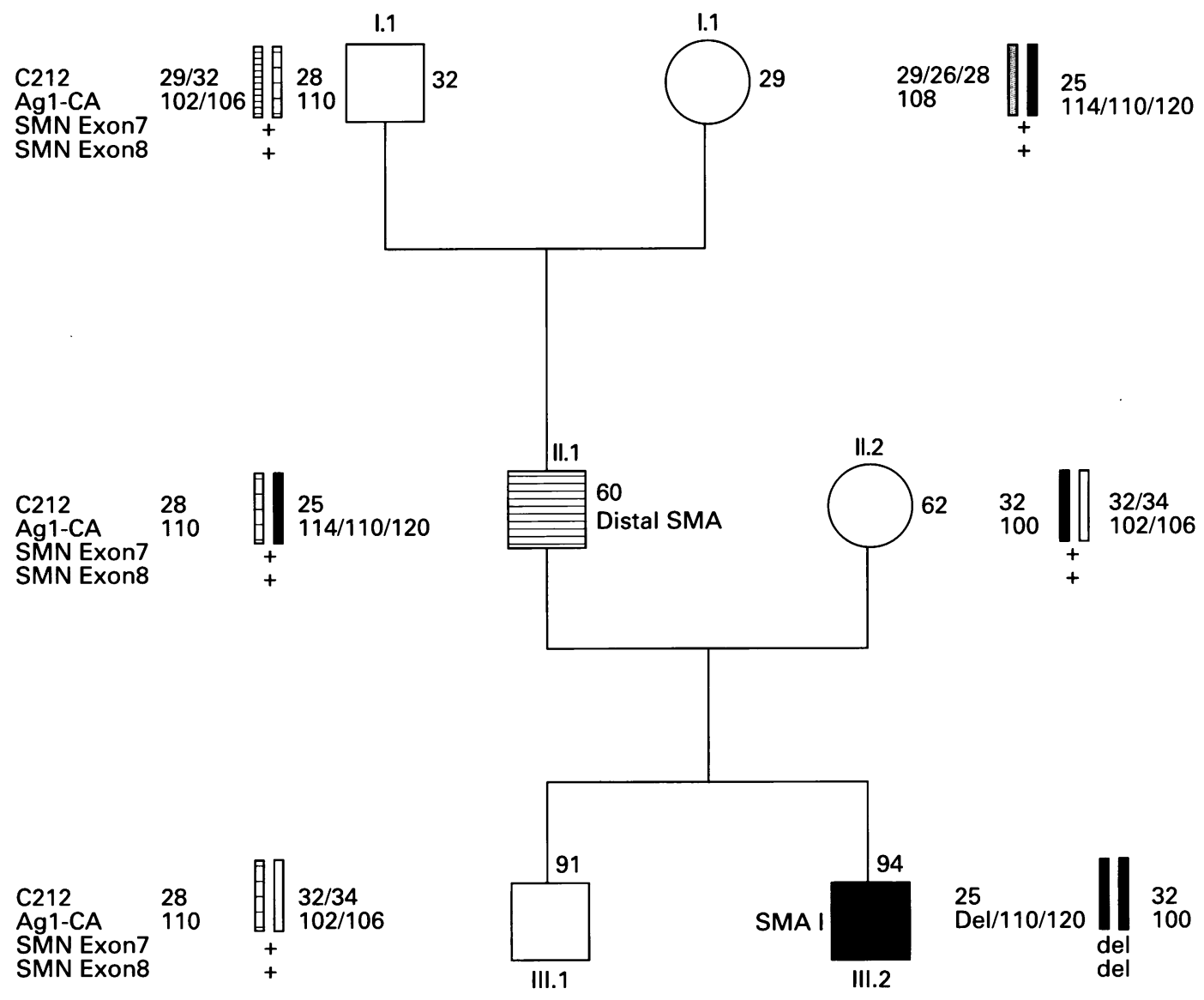

Figure 1 Pedigree of the family showing the results of molecular genetic investigations. The index patient III. 2 shows a homozygous deletion of exons 7 and 8 of the SMN gene and a de novo deletion of one copy of the multicopy marker Ag1-CA in the paternal haplotype.

Ag1-CA (114/110/120), only two copies (110/ 120) were transmitted to his son (fig 1). This indicated a new mutation in the paternal haplotype involving the SMN gene and one copy of the marker Ag1-CA.

\section{Discussion}

The occurrence of both proximal and distal SMA in the same family has been reported rarely. Boylan and Cornblath ${ }^{9}$ described a family with severe infantile SMA and autosomal dominant distal SMA. The authors suggested that infantile SMA in the child might represent severe expression of the distal SMA gene. ${ }^{9}$ The phenotypic variability was attributed to an allelic or non-allelic modification of disease expression by maternally derived genes.

Harding et $a l^{8}$ observed two cases with Werdnig-Hoffmann disease in the son and the grandchild of a patient with distal SMA. The authors speculated that asymmetrical distal SMA might be the result of biochemically deficient anterior horn cells, but they admitted that the coincidence of two disorders in the same family would not be uncommon considering the relatively high gene frequency of infantile SMA.

After mapping of SMA I-III to the region of 5q11.2-13.3, several candidate genes were discovered. The most important candidate gene in the $5 q 13$ region is the telomeric copy of the

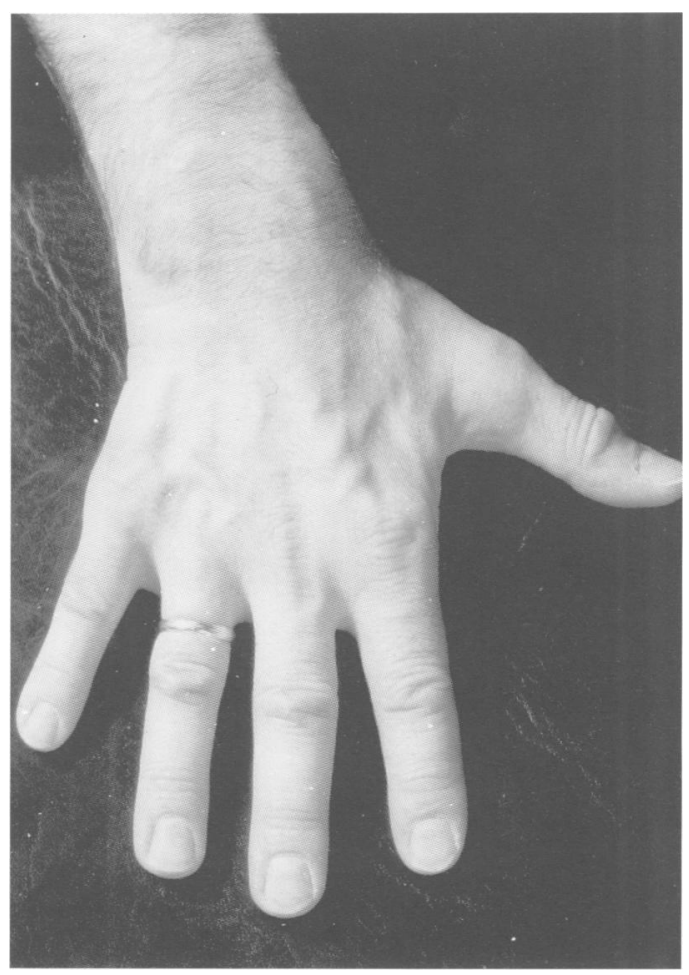

Figure 2 Hands of patient II.1, photographed during attempted spreading of the fingers. There is essentially no lateral movement of the second and third fingers bilaterally. There is marked symmetrical wasting of the interosseous muscles. 
SMN gene, which is homozygously deleted in $98 \%$ of patients with SMA type I. In most cases the deletion affects exons 7 and 8. Deletions are detectable by $\mathrm{SSCP}^{3}$ or restriction fragment length polymorphism analysis ${ }^{10}$ only in a homozygous state. Deletion screening cannot distinguish between heterozygotes and normal controls. Unlike that of the son with infantile SMA (III.2), the SMN gene of the father (II.1) with distal SMA was not homozygously deleted. However, we were unable to show by direct molecular testing whether the parents of the affected child were heterozygous for the SMN deletion or not.

Several multicopy markers have been identified in close proximity to the SMN gene. ${ }^{11}$ These markers, Ag1-CA and C212, were found to be completely deleted on one parental chromosome in about $15 \%$ of severe type I SMA patients, while only exceptionally did type II and III patients show deletions. ${ }^{12}{ }^{13}$ These are always inherited large scale deletions. In addition, the loss of single copies was seen in patients, which can be regarded as de novo events if the transmitting parent still carries a complete set of alleles. ${ }^{12} 13$

Evidence that the father is not heterozygous for deletions in the $5 \mathrm{q} 13$ region was shown by indirect genotype analysis with the multicopy marker Ag1-CA, which lies at the 5' end of the SMN gene. He had three copies, while his affected son had only two. A de novo mutation in the paternal haplotype occurred, involving the SMA region including the SMN gene. These results point towards the assumption that the father does not carry a mutation of the SMN gene. His disease, distal spinal muscular atrophy, is probably not caused by an SMN gene deletion. It can therefore be concluded that his condition is unrelated to the disease in his son.

The combination of autosomal recessive SMA in a child and distal SMA of adult onset in his father is probably caused by the coincidence of different genetic mechanisms.

1 International SMA Collaboration.Workshop Report. Neuromusc Disord 1992;2:423-8.

2 Zerres $K$, Rudnik-Schöneborn S. Natural history in proximal spinal muscular atrophy (SMA) - clinical analysis proximal spinal muscular atrophy (SMA) - clinical analysis of 445 patients and suggestions for a modification

3 Lefebvre S, Bürglen L, Reboullet S, et al. Identification and characterisation of a spinal muscular atrophy determing gene. Cell 1995;80:155-65.

4 Rodrigues NR, Owen N, Talbot K, Ignatius J, Dubowitz V, Davies KE. Deletions in the survival motor neuron gene on $5 \mathrm{q} 13$ in autosomal recessive spinal muscular atrophy. Hum Mol Genet 1995;4:631-4.

5 Hahnen E, Forkert R, Marke C, et al. Molecular analysis of candidate genes on chromosome $5 \mathrm{q} 13$ in autosomal recessive spinal muscular atrophy: evidence of homozygous sive spinal muscular atrophy. evidence of homozygous Mol Genet 1995;4:1927-33.

6 Pearn J, Hudgson P. Distal spinal muscular atrophy, a clini$\mathrm{cal}$ and genetic study of 8 kindreds. $f$ Neurol $\mathrm{Sc}$ 1980;43:183-91.

7 Christodoulou K, Kyriakidis T, Hristova AH, et al. Mapping of a distal form of spinal muscular atrophy with upper limb predominance to chromosome 7p. Hum Mol Genet 1995;4 1629-32.

8 Harding AE, Bradbury PG, Murray NMF. Chronic asymmetrical spinal muscular atrophy. $\mathcal{f}$ Neurol $\mathrm{Sci}$ 1983;59:69-83.

9 Boylan KB, Cornblath DR. Werdnig-Hoffmann disease and chronic distal spinal muscular atrophy with apparent autosomal dominant inheritance. Ann Neurol 1992;32:404-7.

10 Van der Steege G, Grootschollen PM, van der Vlies P, Draaijers TG, Osinga J, Scheffer H. PCR-based DNA test Draaijers TG, Osinga J, Scheffer H. PCR-based DNA test
to confirm clinical diagnosis of autosomal recessive spinal to confirm clinical diagnosis of autosomal

11 DiDonato CJ, Morgan K, Carpten JD, et al. Association between Ag1-CA alleles and severity of autosomal recessive proximal spinal muscular atrophy. Am f Hum Genet 1994 55:1218-29

12 Melki J, Lefebvre S, Bürglen L, et al. De novo and inherited deletions of the 5 q13 region in spinal muscular atrophy. Science 1994;264:1474-7.

13 Wirth B, Hahnen E, Morgan K, et al. Allelic association and deletions in autosomal recessive proximal spinal muscular atrophy: association of marker genotype with disease severatrophy: association of marker genotype with disease sever- 